\title{
Waves
}

\section{Laurie Price}

to be in the foreign body as a luxury of clandestine presence so only in my apparent absence which is hidden but shifted from the always there to sometimes comes, sometimes stays, sometimes goes alone to the beach to walk inside the tidal atmosphere and thick air, blowing sand \& seagulls, attributes of a resting place gaze, gazelles, and let the words drift or sift as worlds I'm coming into in a slow repose to hear the sounds caught in their throats as birds as language as bent between the spaces I can imagine if I keep my mouth closed \& just listen

From the collection Mekhtoub, Lyrics \& Invocations 